\title{
The Green rings of pointed tensor categories of finite type
}

\author{
Hua-Lin Huang ${ }^{\mathrm{a}, *}$, Fred Van Oystaeyen ${ }^{\mathrm{b}}$, Yuping Yang ${ }^{\mathrm{a}}$, Yinhuo Zhang ${ }^{\mathrm{c}}$ \\ a School of Mathematics, Shandong University, Jinan 250100, China \\ ${ }^{\mathrm{b}}$ Department of Mathematics and Computer Science, University of Antwerp, Middelheimlaan 1, B-2020 Antwerp, Belgium \\ ${ }^{\mathrm{c}}$ Department WNI, University of Hasselt, Universitaire Campus, 3590 Diepeenbeek, Belgium
}

\section{A R T I C L E I N F O}

\section{Article history:}

Received 3 January 2013

Received in revised form 18 May 2013

Available online 22 June 2013

Communicated by C.A. Weibel

\section{A B S T R A C T}

In this paper, we compute the Clebsch-Gordan formulae and the Green rings of connected pointed tensor categories of finite type.

(C) 2013 Elsevier B.V. All rights reserved.

MSC: 19A22; 18D10; 16G20

\section{Introduction}

Throughout the paper, $k$ is an algebraically closed field of characteristic zero, and (co)algebras, (co)modules, categories, etc. are over $k$. Concepts and notations about tensor categories are adopted from [7].

Let $\mathcal{C}$ be a tensor category, that is, a locally finite abelian rigid monoidal category in which the neutral object is simple. Note that the Jordan-Hölder theorem and the Krull-Schmidt theorem hold in $\mathcal{C}$. For each $M \in \mathcal{C}$, let $[M]$ denote the associated iso-class. The Green ring $\mathcal{G} \mathcal{R}(\mathcal{C})$ of $\mathcal{C}$ is the ring with generators the iso-classes $[M]$ of $\mathcal{C}$, and relations

$$
[M]+[N]=[M \oplus N], \quad[M] \cdot[N]=[M \otimes N] .
$$

By the Krull-Schmidt theorem, the ring $g \mathcal{R}(\mathcal{C})$ is a free $\mathbb{Z}$-module, and the set of indecomposable iso-classes of $\mathcal{C}$ forms a basis. When $\mathcal{C}=H$-mod, where $H$ is a Hopf algebra, the $\operatorname{ring} g \mathcal{R}(\mathcal{C})$ is also called the Green ring of $H$.

The Green ring $g \mathcal{R}(\mathcal{C})$ provides a convenient way of organizing information about direct sums and tensor products of $\mathcal{C}$. When $\mathcal{C}$ is the category of modular representations of a finite group, the ring $q \mathcal{R}(\mathcal{C})$ was investigated systematically for the first time by J.A. Green [9], hence the notion Green ring is widely used in the literature. Green rings have played an important role in the modular representation theory; see [2] and references therein.

Without a doubt, Green rings should be equally important in the study of those tensor categories of (co)modules over (co)quasi-Hopf algebras (or quasi-quantum groups) which are not semisimple; see [7,8]. However, so far there are not many results obtained about the Green rings of such tensor categories due to the obvious complexity. In [16], the Green ring (called the representation ring there) of the quantum double of a finite group was described by a direct sum decomposition. In a subsequent work [17], the Green ring of the twisted quantum double of a finite group was considered and some results about the Grothendieck ring, that is the quotient of the Green ring by the ideal of short exact sequences, were obtained. Recently, the Green rings of the Taft algebras and the generalized Taft algebras were presented by generators and relations in $[3,14]$ respectively.

The aim of this paper is to compute the Clebsch-Gordan formulae and to present the Green rings of pointed tensor categories of finite type as classified in [13]. Such tensor categories are actually the comodule categories of some pointed

\footnotetext{
* Corresponding author.

E-mail addresses: hualin@sdu.edu.cn (H.-L. Huang), fred.vanoystaeyen@ua.ac.be (F. Van Oystaeyen), yupingyang@mail.sdu.edu.cn (Y. Yang), yinhuo.zhang@uhasselt.be (Y.Zhang).
} 
coquasi-Hopf algebras of finite corepresentation type, or equivalently the module categories of some elementary quasi-Hopf algebras of finite representation type, in which there are only finitely many iso-classes of indecomposable objects, and as such their Green rings are suitable to study. Among them, the module categories of the Taft and generalized Taft algebras are particular examples. In addition, these tensor categories can be presented by quiver representations and we can take full advantage of the handy quiver techniques (see e.g. [1,18]) for the computations. In Section 2, we recall some necessary facts about pointed tensor categories of finite type. The Clebsch-Gordan formulae for such tensor categories are computed in Section 3. Finally in Section 4, the Green rings are determined. The results obtained here extend those in $[5,3,14]$ to a much greater range in a unified way.

\section{Pointed tensor categories of finite type}

In this section, we recall pointed tensor categories of finite type and their presentations via quiver representations.

2.1. Let $n>1$ be a positive integer and $C_{n}=\left\langle g \mid g^{n}=1\right\rangle$ the cyclic group of order $n$. By $Z_{n}$ we denote the cyclic quiver with vertices indexed by $C_{n}$ and with arrows $a_{i}: g^{i} \rightarrow g^{i+1}$ where the indices $i$ are understood as integers modulo $n$. Let $p_{i}^{l}$ denote the path $a_{i+l-1} \cdots a_{i+1} a_{i}$ of length $l$. The $k$-span of $\left\{p_{i}^{l} \mid 0 \leq i \leq n-1, l \geq 0\right\}$ is called the associated path space of the quiver $Z_{n}$, and denoted by $k Z_{n}$.

From now on, let $m^{\prime}$ denote the remainder of the division of the integer $m$ by $n$. There is a natural coalgebra structure on $k Z_{n}$ with coproduct and counit given by

$$
\Delta\left(p_{i}^{l}\right)=\sum_{m=0}^{l} p_{(i+m)^{\prime}}^{l-m} \otimes p_{i}^{m}, \quad \varepsilon\left(p_{i}^{l}\right)=\delta_{l, 0}= \begin{cases}1, & l=0 ; \\ 0, & \text { otherwise. }\end{cases}
$$

This is the so-called path coalgebra of the quiver $Z_{n}$.

2.2. The quiver $Z_{n}$ is in fact a Hopf quiver in the sense of [6]. By [10], there exist graded coquasi-Hopf algebra, also known as Majid algebra, structures on the path coalgebra $k Z_{n}$. Moreover, the graded coquasi-Hopf structures are parameterized by some set of 1-dimensional projective representation of $C_{n}$; see [11] for a general theorem.

Explicit graded coquasi-Hopf structures on $k Z_{n}$ are given in [13]. To state this result, first we need to fix some notations. For any $\hbar \in k$, define $m_{\hbar}=1+\hbar+\cdots+\hbar^{m-1}$ and $m ! \hbar=1_{\hbar} \cdots m_{\hbar}$. The Gaussian binomial coefficient is defined by $\left(\begin{array}{c}m+n \\ m\end{array}\right)_{h}:=\frac{(m+n) ! \hbar}{m ! \hbar n ! \hbar}$. Let $q$ be a primitive $n$-th root of unity. Assume $0 \leq s \leq n-1$ is an integer and $q$ an $n$-th root of $q^{s}$. For each pair $(s, q)$, there is a unique graded coquasi-Hopf algebra $k Z_{n}(s, q)$ on $k Z_{n}$ with multiplication given by

$$
p_{i}^{l} \cdot p_{j}^{m}=\mathbb{q}^{-s j l} q^{-j l} \mathbb{q}_{\mathbb{s}}^{\frac{s\left(i+l^{\prime}\right)\left[m+j-(m+j)^{\prime}\right]}{n}}\left(\begin{array}{c}
l+m \\
l
\end{array}\right)_{\mathbb{q}^{-s} q^{-1}} p_{(i+j)^{\prime}}^{l+m} .
$$

Let $d$ be the order of $q$. Clearly, $d \mid n$ if $s=0$ and $d=\frac{n^{2}}{\left(s, n^{2}\right)}$ if $1 \leq s \leq n-1$. Let $k Z_{n}(d)$ denote the subcoalgebra of $k Z_{n}$ which as a $k$-space is spanned by $\left\{p_{i}^{l} \mid 0 \leq i \leq n-1,0 \leq l \leq d-1\right\}$. The multiplication (2.1) is closed inside $k Z_{n}(d)$ and it becomes a graded coquasi-Hopf algebra, denoted by $M(n, s, q)$.

2.3. A pointed tensor category of finite type is a tensor category in which there are only finitely many iso-classes of indecomposable objects and whose simple objects are invertible; see [7,13].

By $\mathcal{C}(n, s, q)$ we denote the category of finite-dimensional right $M(n, s, q)$-comodules. It is proved in [13] that any pointed tensor category of finite type consists of finitely many identical components, and the connected component containing the neutral object is equivalent to a deformation of some $\mathcal{C}(n, s, q)$, and see [13, Theorem 4.2 and Corollary 4.4] for a full description.

In this paper we focus on the Clebsch-Gordan formulae and the Green ring of $\mathcal{C}(n, s, q)$. The results can be easily extended to the non-connected case. Now we give an explicit presentation of $\mathcal{C}(n, s, q)$ by quiver representations. Recall that a representation of the quiver $Z_{n}$ is a collection $V=\left(V_{i}, T_{i}\right)_{0 \leq i \leq n-1}$ where $V_{i}$ is a vector space corresponding to the vertex $g^{i}$ and $T_{i}: V_{i} \rightarrow V_{i+1}$ is a linear map corresponding to the arrow $a_{i}$. Given a path $p_{i}^{l}$, we define a corresponding linear map $T_{i}^{l}$ as follows. If $l=0$, then put $T_{i}^{0}=\operatorname{Id}_{V_{i}}$. If $l>0$, put $T_{i}^{l}=T_{i+l-1} \cdots T_{i+1} T_{i}$. The category $\mathcal{C}(n, s, q)$ consists of the representations $V$ of $Z_{n}$ such that $T_{i}^{l}=0$ whenever $l \geq d$.

The indecomposable objects of $\mathcal{C}(n, s, q)$ can be described as follows. Assume $0 \leq i \leq n-1$ and $0 \leq e \leq d-1$. Let $V(i, e)$ be a vector space of dimension $e+1$ with a basis $\left\{v_{m}^{i}\right\}_{0 \leq m \leq e} . V(i, e)$ is made into a representation of $Z_{n}$ by putting $V(i, e)_{j}$ the $k$-span of $\left\{v_{m}^{i} \mid(i+m)^{\prime}=j\right\}$ and letting $T(i, e)_{j}$ maps $v_{m}^{i}$ to $v_{m+1}^{i}$ if $(i+m)^{\prime}=j$. Here the vector $v_{e+1}^{i}$ is understood as 0 by convention. Clearly $V(i, e)$ is an object in $\mathcal{C}(n, s, q)$ and $\{V(i, e)\}_{0 \leq i \leq n-1,0 \leq e \leq d-1}$ is a complete set of iso-classes of indecomposable objects of $\mathcal{C}(n, s, q)$.

2.4. For later computations, we need to know how the quiver representation $V(i, e)$ is viewed as a right $M(n, s, q)$-comodule. Let

$$
\delta: V(i, e) \rightarrow V(i, e) \otimes M(n, s, q)
$$


denote the comodule structure map, then

$$
\delta\left(v_{m}^{i}\right)=\sum_{x=m}^{e} v_{x}^{i} \otimes p_{(i+m)^{\prime}}^{x-m} .
$$

For any two indecomposable objects $V(i, e)$ and $V(j, m)$ of $\mathcal{C}(n, s, q)$, the comodule structure map of their tensor product $V(i, e) \otimes V(j, m)$ is given by

$$
\delta\left(v_{a}^{i} \otimes v_{b}^{j}\right)=\sum_{x=s}^{e} \sum_{y=t}^{m} C_{(i, a)(j, b)}^{(i, x)(j, y)} v_{x}^{i} \otimes v_{y}^{j} \otimes p_{(i+a+j+b)^{\prime}}^{x+y-a-b},
$$

where $C_{(i, a)(j, b)}^{(i, x)(j, y)}$ is the coefficient of the product $p_{(i+a)^{\prime}}^{x-a} \cdot p_{(j+b)^{\prime}}^{y-b}$ as given in (2.2). It is clear that $C_{(i, a)(j, b)}^{(i, x)(j, y)}=0$ whenever $x+y-a-b \geq d$.

\section{The Clebsch-Gordan formulae}

In this section we compute the complete decomposition of $V(i, e) \otimes V(j, f)$ with the help of some techniques from quivers and their representations.

\subsection{Reduction of $V(i, e) \otimes V(j, f)$ to $V(0, e) \otimes V(0, f)$}

First of all, we list some useful facts of indecomposable comodules of $M(n, s, q)$.

\section{Lemma 3.1.}

$$
\begin{aligned}
& V(0,0) \otimes V(i, e) \cong V(i, e)=V(i, e) \otimes V(0,0), \\
& V(1,0)^{\otimes l}=V\left(l^{\prime}, 0\right), \quad V(1,0)^{\otimes n}=V(0,0), \\
& V(1,0) \otimes V(i, e)=V\left((i+1)^{\prime}, e\right)=V(i, e) \otimes V(1,0), \\
& \forall 0 \leq i \leq n-1,0 \leq e \leq d-1 .
\end{aligned}
$$

Proof. (3.1) and (3.2) are easy by definition. We prove only (3.3). Let $\left\{v_{0}^{1}\right\}$ and $\left\{v_{k}^{i}\right\}_{0 \leq k \leq e}$ be respectively a basis of $V(1,0)$ and $V(i, e)$. Then $\left\{v_{0}^{1} \otimes v_{k}^{i}\right\}_{0 \leq k \leq e}$ is a basis of $V(1,0) \otimes V(i, e)$ and the comodule structure is given by

$$
\delta\left(v_{0}^{1} \otimes v_{k}^{i}\right)=\sum_{x=k}^{e} C_{x} v_{0}^{1} \otimes v_{x}^{i} \otimes p_{(i+k+1)^{\prime}}^{x-k}
$$

Note that $C_{x} \neq 0$ by (2.4). Now view $V(1,0) \otimes V(i, e)$ as a representation of $Z_{n}$. (2.4) implies that $v_{0}^{1} \otimes v_{k}^{i}$ should lie in the vector space attached to the vertex $g^{(i+k+1)^{\prime}}$. On the other hand, by $(3.2) V(1,0)$ is invertible. It follows that $V(1,0) \otimes V(i, e)$ is indecomposable. Now by comparing it with the complete set of iso-classes of indecomposable comodules of $M(n, s, q)$, it is easy to see that $V(1,0) \otimes V(i, e)=V\left((i+1)^{\prime}, e\right)$. Similarly, we have $V(i, e) \otimes V(1,0)=V\left((i+1)^{\prime}, e\right)$.

Thanks to the lemma, we can reduce the decomposition of $V(i, e) \otimes V(j, f)$ to $V(0, e) \otimes V(0, f)$.

\section{Corollary 3.2.}

$$
\begin{aligned}
& V(i, e)=V(1,0)^{\otimes i} \otimes V(0, e)=V(0, e) \otimes V(1,0)^{\otimes i}, \\
& V(i, e) \otimes V(j, f)=V(1,0)^{\otimes(i+j)} \otimes V(0, e) \otimes V(0, f) .
\end{aligned}
$$

Proof. Follows easily from Lemma 3.1.

\subsection{Decomposition of $V(0, e) \otimes V(0, f)$ at vertices}

In the rest of this section, we write $V=V(0, e) \otimes V(0, f)$ for brevity. View $V$ as a representation of $Z_{n}$ and let $V_{i}$ denote the corresponding subspace attached to the vertex $g^{i}$. Note that $V=\oplus_{i=0}^{n-1} V_{i}$ is a vector space. This is the so-called decomposition at vertices, providing essential information for us to detect its direct summands in the next subsection.

With the same notations as in Section 2.3, we have a basis $\left\{v_{k}^{0} \otimes v_{l}^{0}\right\}_{0 \leq k \leq e, 0 \leq l \leq f}$ of $V$. By (2.4), it is clear that

$$
V_{i}=\mathbb{C}\left\{v_{k}^{0} \otimes v_{l}^{0} \mid(k+l)^{\prime}=i, 0 \leq k \leq e, 0 \leq l \leq f\right\}
$$

and hence its dimension is given by

$$
\operatorname{dim} V_{i}=\left|\left\{(k, l) \in \mathbb{Z} \times \mathbb{Z}:(k+l)^{\prime}=i, 0 \leq k \leq e, 0 \leq l \leq f\right\}\right| .
$$


In order to give an explicit formula for $\operatorname{dim} V_{i}$, we need the following technical lemma.

Lemma 3.3. For integers $0 \leq \alpha, \beta \leq n-1$, let

$$
N_{(\alpha, \beta)}^{k}=\left|\left\{(x, y) \in \mathbb{Z} \times \mathbb{Z}:(x+y)^{\prime}=k, 0 \leq x \leq \alpha, 0 \leq y \leq \beta\right\}\right| .
$$

If $\alpha \leq \beta$ and $\alpha+\beta \leq n-1$, then

$$
N_{(\alpha, \beta)}^{k}= \begin{cases}k+1, & 0 \leq k \leq \alpha, \\ \alpha+1, & \alpha \leq k \leq \beta, \\ \alpha+\beta+1-k, & \beta \leq k \leq \alpha+\beta ;\end{cases}
$$

if $\alpha \leq \beta$ and $\alpha+\beta \geq n$, set $\gamma=\alpha+\beta-n+1$, then

$$
N_{(\alpha, \beta)}^{k}= \begin{cases}\gamma+1, & 0 \leq k \leq \gamma, \\ k+1, & \gamma \leq k \leq \alpha, \\ \alpha+1, & \alpha \leq k \leq \beta, \\ \alpha+\beta+1-k, & \beta \leq k \leq n-1 ;\end{cases}
$$

if $\alpha>\beta$ and $\alpha+\beta \leq n-1$, then

$$
N_{(\alpha, \beta)}^{k}= \begin{cases}k+1, & 0 \leq k \leq \beta, \\ \beta+1, & \beta \leq k \leq \alpha, \\ \beta+\alpha+1-k, & \alpha \leq k \leq \alpha+\beta ;\end{cases}
$$

if $\alpha>\beta$ and $\alpha+\beta \geq n$, set $\gamma$ as above, then

$$
N_{(\alpha, \beta)}^{k}= \begin{cases}\gamma+1, & 0 \leq k \leq \gamma, \\ k+1, & \gamma \leq k \leq \beta, \\ \beta+1, & \beta \leq k \leq \alpha, \\ \alpha+\beta+1-k, & \alpha \leq k \leq n-1 .\end{cases}
$$

Proof. By direct computation.

\section{Lemma 3.4}

$$
\operatorname{dim} V_{i}=\frac{e f-e^{\prime} f^{\prime}}{n}+\frac{e+f-e^{\prime}-f^{\prime}}{n}+N_{\left(e^{\prime}, f^{\prime}\right)}^{i} .
$$

Proof. Write $e=\alpha n+e^{\prime}, f=\beta n+f^{\prime}$ and

$$
\{(x, y): 0 \leq x \leq e, 0 \leq y \leq f\}=U_{1} \cup U_{2} \cup U_{3} \cup U_{4}
$$

where

$$
\begin{aligned}
& U_{1}=\{(x, y): 0 \leq x \leq \alpha n-1,0 \leq y \leq \beta n-1\} \\
& U_{2}=\{(x, y): \alpha n \leq x \leq e, 0 \leq y \leq \beta n-1\} \\
& U_{3}=\{(x, y): 0 \leq x \leq \alpha n-1, \beta n \leq y \leq f\} \\
& U_{4}=\{(x, y): \alpha n \leq x \leq e, \beta n \leq y \leq f\}
\end{aligned}
$$

By easy computation and Lemma 3.3 one has:

$$
\begin{aligned}
& \left|\left\{(x, y) \in U_{1}:(x+y)^{\prime}=i\right\}\right|=n \alpha \beta, \\
& \left|\left\{(x, y) \in U_{2}:(x+y)^{\prime}=i\right\}\right|=\beta\left(e^{\prime}+1\right), \\
& \left|\left\{(x, y) \in U_{3}:(x+y)^{\prime}=i\right\}\right|=\alpha\left(f^{\prime}+1\right), \\
& \left|\left\{(x, y) \in U_{4}:(x+y)^{\prime}=i\right\}\right|=N_{\left(e^{\prime}, f^{\prime}\right)}^{i} .
\end{aligned}
$$

Now we obtain:

$$
\begin{aligned}
\operatorname{dim} V_{i} & =n \alpha \beta+\alpha f^{\prime}+e^{\prime} \beta+\alpha+\beta+N_{\left(e^{\prime}, f^{\prime}\right)}^{i} \\
& =\frac{e f-e^{\prime} f^{\prime}}{n}+\frac{e+f-e^{\prime}-f^{\prime}}{n}+N_{\left(e^{\prime}, f^{\prime}\right)}^{i} .
\end{aligned}
$$




\subsection{The Clebsch-Gordan formulae}

The main objective of this subsection is to compute the decomposition of $V=V(0, e) \otimes V(0, f)$ which will easily lead to the general Clebsch-Gordan formulae for $\mathcal{C}=\mathcal{C}(n, s, q)$.

By $S_{i}(U)$ we denote the number of direct summands of the form $V(i$, ?) that appear in the decomposition of $U \in \mathcal{C}$. As in Section 2.3, we view $U$ as a representation of the quiver $Z_{n}$ and let $T_{i}: U_{i} \rightarrow U_{i+1}$ be the linear map attached to the arrow $a_{i}$. Write

$$
U_{i}^{\prime}=\operatorname{ker} T_{i}=\left\{x \in U_{i}: T_{i}(x)=0\right\} .
$$

\section{Lemma 3.5.}

$$
S_{i}(U)=\operatorname{dim} U_{i}-\operatorname{dim} U_{(i-1)^{\prime}}+\operatorname{dim} U_{(i-1)^{\prime}}^{\prime} .
$$

Proof. If $U=V(i, l)$, then one observes that

$$
\operatorname{dim} U_{i}-\operatorname{dim} U_{(i-1)^{\prime}}+\operatorname{dim} U_{(i-1)^{\prime}}^{\prime}=1=S_{i}(U) ;
$$

if $U=V(m, l)$ with $m \neq i$, one has

$$
\operatorname{dim} U_{i}-\operatorname{dim} U_{(i-1)^{\prime}}+\operatorname{dim} U_{(i-1)^{\prime}}^{\prime}=0=S_{i}(U) .
$$

In general, if $U=\oplus V(j, l)$, then $U_{i}=\oplus V(j, l)_{i}$ and $U_{i}^{\prime}=\oplus V(j, l)_{i}^{\prime}$. Now we have

$$
\begin{aligned}
S_{i}(U) & =\sum S_{i}(V(j, l)) \\
& =\sum\left[\operatorname{dim} V(j, l)_{i}-\operatorname{dim} V(j, l)_{(i-1)^{\prime}}+\operatorname{dim} V(j, l)_{i}^{\prime}\right] \\
& =\operatorname{dim} U_{i}-\operatorname{dim} U_{(i-1)^{\prime}}+\operatorname{dim} U_{(i-1)^{\prime}}^{\prime} .
\end{aligned}
$$

Now we come back to $V=V(0, e) \otimes V(0, f)$. To compute $S_{i}(V)$, it suffices to determine $\operatorname{dim} V_{i}^{\prime}$.

\section{Proposition 3.6.}

$$
\operatorname{dim} V_{i}^{\prime}= \begin{cases}\left|\left\{j: f-e \leq j \leq f,(e+j)^{\prime}=i\right\}\right|, & \text { if } e \leq f \\ \left|\left\{j: e-f \leq j \leq e,(e+j)^{\prime}=i\right\}\right|, & \text { if } e>f\end{cases}
$$

Proof. Clearly the two cases are symmetric, so it is enough to prove one of them. Assume $e \leq f$. Take the bases $\left\{v_{l}^{0}\right\}_{0 \leq l \leq e}$ and $\left\{v_{m}^{0}\right\}_{0 \leq m \leq f}$ for $V(0, e)$ and $V(0, f)$ respectively as before. Assume $u \in V_{i}^{\prime}$. We can write

$$
u=\sum_{(l+m)^{\prime}=i} \lambda_{l m} v_{l}^{0} \otimes v_{m}^{0}=\sum_{x} \sum_{l+m=n x+i} \lambda_{l m} v_{l}^{0} \otimes v_{m}^{0} .
$$

Let $u_{x}=\sum_{l+m=n x+i} \lambda_{l m} v_{l}^{0} \otimes v_{m}^{0}$. Then it is clear that $T(u)=0$ if and only if $T\left(u_{x}\right)=0, \forall x$. Rewrite $u_{x}=\lambda_{1} v_{l_{1}}^{0} \otimes v_{m_{1}}^{0}+\cdots+$ $\lambda_{y} v_{l_{y}}^{0} \otimes v_{m_{y}}^{0}$ with $\lambda_{k} \neq 0, \forall k$, such that $l_{1}>\cdots>l_{y}$ and hence $m_{1}<\cdots<m_{y}$. Recall that:

$$
T_{i}\left(v_{x}^{0} \otimes v_{y}^{0}\right)=\mathbb{q}^{-s y} q^{-y} v_{x+1}^{0} \otimes v_{y}^{0}+\mathbb{q}^{\frac{s x\left[1+y-(1+y)^{\prime}\right]}{n}} v_{x}^{0} \otimes v_{y+1}^{0}
$$

if $x<e, y<f$. It follows that if $l_{1} \neq e$, we have:

$$
T_{i}\left(u_{x}\right)=\lambda_{1} \Phi^{-s m_{1}} q^{-m_{1}} v_{l_{1}+1}^{0} \otimes v_{m_{1}}^{0}+\sum \text { other terms } \neq 0 .
$$

This forces $l_{1}=e$ if $T_{i}\left(u_{x}\right)=0$. By a similar argument one has $m_{y}=f$ if $T_{i}\left(u_{x}\right)=0$. Clearly it is necessary that $l_{1} \leq f$ and the subindex of the first factor of the last term $e+l_{1}-f \geq 0$. That is, $f-e \leq l_{1} \leq f$.

By appropriate rescaling $u_{x}$ is of the following form

$$
u_{x}=v_{e}^{0} \otimes v_{j}^{0}+a_{1} v_{e-1}^{0} \otimes v_{j+1}^{0}+\cdots+a_{(f-j)} v_{e+j-f}^{0} \otimes v_{f}^{0}
$$

with $j=n x+i-e$ and $f-e \leq j \leq f$. By imposing the condition $T_{i}\left(u_{x}\right)=0$ one can solve out a unique set of the $a_{i}$ 's by recursion. As the explicit solution will not be used in later argument, we omit the calculation.

Now we have proved that $V_{i}^{\prime}$ is spanned by elements like $u_{x}$. Clearly the $u_{x}$ 's are linearly independent. Therefore, the dimension of $V_{i}^{\prime}$ is equal to the number of such $u_{x}$ 's, which is exactly $\left|\left\{j: f-e \leq j \leq f,(e+j)^{\prime}=i\right\}\right|$.

With this preparation, now we can compute $S_{i}=S_{i}(V)$. 
Proposition 3.7. Suppose $e=\alpha n+e^{\prime}, f=\beta n+f^{\prime}$. Then we have:

$$
S_{i}= \begin{cases}\alpha+1, & e \leq f, 0 \leq i \leq e^{\prime} \\ \alpha, & e \leq f, e^{\prime}<i \leq n-1 ; \\ \beta+1, & e>f, 0 \leq i \leq f^{\prime} \\ \beta, & e>f, f^{\prime}<i \leq n-1 .\end{cases}
$$

Proof. We prove only the cases with $e \leq f$. Write $d^{i}$ for $\operatorname{dim} V_{i}^{\prime}$. Assume first $e^{\prime}+f^{\prime} \leq n-1$ and $e^{\prime} \leq f^{\prime}$.

If $e^{\prime}+f^{\prime}<n-1$, by applying Lemmas 3.3-3.5 we have:

$$
S_{i}= \begin{cases}d^{i-1}+1, & 0 \leq i \leq e^{\prime} ; \\ d^{i-1}, & e^{\prime}+1 \leq i \leq f^{\prime} ; \\ d^{i-1}-1, & f^{\prime}+1 \leq i \leq e^{\prime}+f^{\prime}+1 ; \\ d^{i-1}, & e^{\prime}+f^{\prime}+2 \leq i \leq n-1 .\end{cases}
$$

Further application of Proposition 3.6 leads to:

$$
S_{i}= \begin{cases}\alpha+1, & 0 \leq i \leq e^{\prime} ; \\ \alpha, & e^{\prime}+1 \leq i \leq f^{\prime} \\ \alpha, & f^{\prime}+1 \leq i \leq e^{\prime}+f^{\prime}+1 \\ \alpha, & e^{\prime}+f^{\prime}+2 \leq i \leq n-1 .\end{cases}
$$

Note that if $e^{\prime}+f^{\prime}+1=n-1$, the last case actually vanishes.

If $e^{\prime}+f^{\prime}=n-1$, then by Lemmas 3.3-3.5 we have:

$$
S_{i}= \begin{cases}d^{i-1}+1, & i=0 \\ d^{i-1}, & 1 \leq i \leq e^{\prime} \\ d^{i-1}-1, & e^{\prime}+1 \leq i \leq f^{\prime} \\ d^{i-1}, & f^{\prime}+1 \leq i \leq e^{\prime}+f^{\prime}=n-1 .\end{cases}
$$

Following Proposition 3.6 we have:

$$
S_{i}= \begin{cases}\alpha+1, & i=0 \\ \alpha+1, & 1 \leq i \leq e^{\prime} \\ \alpha, & e^{\prime}+1 \leq i \leq f^{\prime} \\ \alpha, & f^{\prime}+1 \leq i \leq n-1 .\end{cases}
$$

Therefore,

$$
S_{i}= \begin{cases}\alpha+1, & 0 \leq i \leq e^{\prime} \\ \alpha, & e^{\prime}+1 \leq i \leq n-1 .\end{cases}
$$

The remaining three cases with $e^{\prime}+f^{\prime} \leq n-1, e^{\prime}>f^{\prime} ; e^{\prime}+f^{\prime} \geq n, e^{\prime} \leq f^{\prime}$ and $e^{\prime}+f^{\prime} \geq n, e^{\prime}>f^{\prime}$ respectively can be dealt with by the same process, so the detail is omitted.

From now on, we shall understand $V\left(i\right.$, ?) as $V\left(i^{\prime}\right.$, ?) if $i \geq n$. With this notation, we have the following nice form for the complete decomposition of $V=V(0, e) \otimes V(0, f)$.

\section{Corollary 3.8.}

$$
V= \begin{cases}V(0, ?) \oplus V(1, ?) \oplus \cdots \oplus V(e, ?), & \text { if } e \leq f \\ V(0, ?) \oplus V(1, ?) \oplus \cdots \oplus V(f, ?), & \text { otherwise }\end{cases}
$$

Proof. Direct consequence of Proposition 3.7.

Now we are in the position to give the main result of this section.

Theorem 3.9. If $e+f \leq d-1$, then

$$
\begin{aligned}
& V= \begin{cases}\bigoplus_{i=0}^{e} V(i, e+f-2 i), & \text { if } e \leq f ; \\
\bigoplus_{i=0}^{f} V(i, e+f-2 i), & \text { otherwise. }\end{cases} \\
& \text { If } e+f \geq d \text {, set } \gamma=e+f-d+1 \text {, then } \\
& V= \begin{cases}\bigoplus_{i=0}^{\gamma} V(i, d-1) \oplus \bigoplus_{j=\gamma+1}^{e} V(j, e+f-2 j), & \text { if } e \leq f ; \\
\bigoplus_{i=0}^{\gamma} V(i, d-1) \oplus \bigoplus_{j=\gamma+1}^{f} V(j, e+f-2 j), & \text { otherwise. }\end{cases}
\end{aligned}
$$


Proof. As before, it is enough to show the case with $e \leq f$. We prove by induction on $e$. First of all we consider the case with $e+f \leq d-1$. When $e=0$, the claim is trivial. Consider $e=1$. By Corollary 3.8, we know $V(0,1) \otimes V(0, f)=V(0, l) \oplus V(1, m)$ for some $l, m$.

Now we view $V$ as a quiver representation and keep the same notations as in Section 2. Note that, under our assumptions, $f<d-1, T_{1}^{f}(V) \neq 0$ and $T_{1}^{f+1}(V)=0$. It follows that $m \leq f$ and so $T_{0}^{f}(V(1, m))=0$. On the other hand, $T_{0}^{f+1}(V) \neq 0$ and $T_{0}^{f+2}(V)=0$ imply that $T_{0}^{f+1}(V(0, l)) \neq 0$ and $T_{0}^{f+2}(V(0, l))=0$. That is to say, $l=f+1$. By comparing the dimensions, we get $m=f-1$. Thus we have:

$$
V(0,1) \otimes V(0, f)=V(0, f+1) \oplus V(1, f-1) .
$$

Similarly, one can prove that:

$$
V(0, f) \otimes V(0,1)=V(0, f+1) \oplus V(1, f-1) .
$$

In particular, we obtain that:

$$
V(0,1) \otimes V(0, f)=V(0, f) \otimes V(0,1) .
$$

Now we assume $1<e \leq f$ and $e+f \leq d-1$. By (3.21) we have

$$
[V(0, e) \oplus V(1, e-2)] \otimes V(0, f)=V(0, e-1) \otimes V(0,1) \otimes V(0, f) .
$$

By the induction hypothesis, we have

$$
\begin{aligned}
V(1, e-2) \otimes V(0, f) & =V(1,0) \otimes V(0, e-2) \otimes V(0, f) \\
& =\bigoplus_{i=0}^{e-2} V(i+1, e-2+f-2 i),
\end{aligned}
$$

and

$$
\begin{aligned}
V(0, e-1) \otimes V(0,1) \otimes V(0, f) & =V(0, e-1) \otimes[V(0, f+1) \oplus V(1, f-1)] \\
& =[V(0, e-1) \otimes V(0, f+1)] \oplus[(V(1,0) \otimes V(0, e-1) \otimes V(0, f-1)] \\
& =\bigoplus_{i=0}^{e-1} V(i, e+f-2 i) \oplus \bigoplus_{j=0}^{e-1} V(j+1, e+f-2-2 j) .
\end{aligned}
$$

By subtracting the foregoing two identities, we obtain:

$$
V(0, e) \otimes V(0, f)=\bigoplus_{i=0}^{e} V(i, e+f-2 i)
$$

Next we prove the case with $e+f \geq d$. We consider first the case with $f=d-1$. By Corollary 3.8 and by comparing the dimensions and the numbers of direct summands one can easily obtain:

$$
V(0, e) \otimes V(0, d-1)=V(0, d-1) \otimes V(0, e)=\bigoplus_{i=0}^{e} V(i, d-1) .
$$

In the rest of the proof, we can always assume $e \leq f<d-1$, hence $e \leq d-2$.

For the general situation, we split it into three cases. Namely, $e+f=d, e+f=d+1$, and $e+f>d+1$.

If $e+f=d$, by the induction hypothesis we have:

$$
\begin{aligned}
V(1, e-2) \otimes V(0, f) & =V(1,0) \otimes V(0, e-2) \otimes V(0, f) \\
& =\bigoplus_{i=0}^{e-2} V(i+1, e+f-2-2 i)
\end{aligned}
$$

and

$$
\begin{aligned}
V(0, e-1) \otimes V(0,1) \otimes V(0, f) & =[V(0, e-1) \otimes V(0, f+1)] \oplus[V(0, e-1) \otimes V(1, f-1)] \\
& =\bigoplus_{i=0}^{1} V(i, d-1) \oplus \bigoplus_{j=2}^{e-1} V(j, e+f-2 j) \oplus \bigoplus_{k=0}^{e-1} V(k+1, e+f-2-2 k) .
\end{aligned}
$$

In view of (3.23), we obtain the following by subtracting the above two identities:

$$
V(0, e) \otimes V(0, f)=\bigoplus_{i=0}^{1} V(i, d-1) \oplus \bigoplus_{j=2}^{e} V(j, e+f-2 j) .
$$


If $e+f=d+1$, by induction assumption we have

$$
V(1, e-2) \otimes V(0, f)=V(1, d-1) \oplus \bigoplus_{i=1}^{e-2} V(i+1, e+f-2-2 i)
$$

and

$$
\begin{aligned}
V(0, e-1) \otimes V(0,1) \otimes V(0, f)= & {[V(0, e-1) \otimes V(0, f+1)] \oplus[V(0, e-1) \otimes V(1, f-1)] } \\
= & \bigoplus_{i=0}^{2} V(i, d-1) \oplus \bigoplus_{j=3}^{e-1} V(j, e+f-2 j) \\
& \oplus V(1, d-1) \oplus \bigoplus_{k=1}^{e-1} V(k+1, e+f-2-2 k) .
\end{aligned}
$$

Again subtracting the foregoing two identities gives us:

$$
V(0, e) \otimes V(0, f)=\bigoplus_{i=0}^{2} V(i, d-1) \oplus \bigoplus_{j=3}^{e} V(j, e+f-2 j) .
$$

If $e+f>d+1$, then $\gamma=e+f-d+1>2$ and by induction hypothesis we have:

$$
V(1, e-2) \otimes V(0, f)=\bigoplus_{i=0}^{\gamma-2} V(i+1, d-1) \oplus \bigoplus_{j=\gamma-1}^{e-2} V(j, e+f-2-2 j)
$$

and

$$
\begin{aligned}
V(0, e-1) \otimes V(0,1) \otimes V(0, f)= & {[V(0, e-1) \otimes V(0, f+1)] \oplus[V(0, e-1) \otimes V(1, f-1)] } \\
= & \bigoplus_{i=0}^{\gamma} V(i, d-1) \oplus \bigoplus_{j=\gamma+1}^{e-1} V(j, e+f-2 j) \\
& \oplus \bigoplus_{k=0}^{\gamma-2} V(k+1, d-1) \oplus \bigoplus_{l=\gamma-1}^{e-1} V(l+1, e+f-2-2 l) .
\end{aligned}
$$

By subtracting the above two identities, we obtain:

$$
V(0, e) \otimes V(0, f)=\bigoplus_{i=0}^{\gamma} V(i, d-1) \oplus \bigoplus_{j=\gamma+1}^{e} V(j, e+f-2 j) .
$$

Thus the proof is completed.

Now by combining Lemma 3.1 and Theorem 3.9, we arrive at the general Clebsch-Gordan formulae of $\mathcal{C}$.

Theorem 3.10. Let $V=V(i, e) \otimes V(j, f)$. If $e+f \leq d-1$, then

$$
V= \begin{cases}\bigoplus_{k=0}^{e} V(i+j+k, e+f-2 k), & \text { if } e \leq f ; \\ \bigoplus_{k=0}^{f} V(i+j+k, e+f-2 k), & \text { otherwise. }\end{cases}
$$

If $e+f \geq d$, set $\gamma=e+f-d+1$, then

$$
V= \begin{cases}\bigoplus_{k=0}^{\gamma} V(i+j+k, d-1) \oplus \bigoplus_{l=\gamma+1}^{e} V(i+j+l, e+f-2 l), & \text { if } e \leq f ; \\ \bigoplus_{k=0}^{\gamma} V(i+j+k, d-1) \oplus \bigoplus_{l=\gamma+1}^{f} V(i+j+l, e+f-2 l), & \text { otherwise. }\end{cases}
$$

Remark 3.11. As an immediate consequence of Theorem 3.9, we obtain the commutativity of the tensor product in $\mathcal{C}$ :

$$
V(i, e) \otimes V(j, f)=V(j, f) \otimes V(i, e) .
$$

\section{The Green rings}

Denote by $\mathcal{G R}(\mathcal{C})$ the Green ring of $\mathcal{C}=\mathcal{C}(n, s, q)$. So far we know that $\{[V(i, l)]: 0 \leq i \leq n-1,0 \leq l \leq d-1\}$ form a basis of $g \mathcal{R}(\mathcal{C})$ and Theorem 3.9 provides the ring structure with explicit structure constants. The aim of this section is to present the Green ring $\mathcal{G} \mathcal{R}(\mathcal{C})$ by generators and relations.

We start with the following lemma which summarizes some important information of $\mathcal{G} \mathcal{R}(\mathcal{C})$ provided by the previous sections. 
Lemma 4.1. Let 1 denote the identity of $\mathcal{G} \mathcal{R}(\mathcal{C})$. Then we have

(1) $[V(1,0)]^{n}=[V(0,0)]=1$.

(2) $[V(0, l)]=[V(0,1)][V(0, l-1)]-[V(1,0)][V(0, l-2)], \forall 2 \leq l \leq d-1$.

(3) $[V(0,1)][V(0, d-1)]=[V(0, d-1)]+[V(1,0)][V(0, d-1)]$.

Proof. (1) Follows from Lemma 3.1.

(2) Direct consequence of (3.20).

(3) Direct consequence of (3.24).

To present the Green ring $\mathscr{g} \mathcal{R}(\mathcal{C})$, we need the generalized Fibonacci polynomials used in $[3,14]$. Set $f_{1}(x, y)=1$ and $f_{2}(x, y)=y$, then define recursively

$$
f_{i}(x, y)=y f_{i-1}(x, y)-x f_{i-2}(x, y), \quad \forall i>2 .
$$

Lemma 4.2 ([3, Lemma 3.11]). For all $n \geq 2$, we have

$$
f_{n}(x, y)=\sum_{i=0}^{\left[\frac{n-1}{2}\right]}(-1)^{i}\left(\begin{array}{c}
n-1-i \\
i
\end{array}\right) x^{i} y^{n-1-2 i}
$$

Now we have the following expression of element $[V(i, l)]$.

Lemma 4.3. In the ring $g \mathcal{R}(\mathcal{C})$

$$
[V(i, l)]=[V(1,0)]^{i} f_{l+1}([V(1,0)],[V(0,1)]), \quad \forall 0 \leq i \leq n-1, l \leq d-1 .
$$

In particular, $\mathscr{Q} \mathcal{R}(\mathcal{C})$ is generated by $[V(1,0)]$ and $[V(0,1)]$.

Proof. Direct consequence of Lemma 4.1(2) and Lemma 4.2.

Finally we are ready to present the Green ring $g \mathcal{R}(\mathcal{C})$.

Theorem 4.4. Let $\mathbb{Z}[x, y]$ be the polynomial ring over $\mathbb{Z}$ with two variables $x$ and $y$. By $J$ we denote the ideal of $\mathbb{Z}[x, y]$ generated by $\left\{x^{n}-1,(y-x-1) f_{d}(x, y)\right\}$. The Green ring $g \mathcal{R}(\mathcal{C})$ is isomorphic to the quotient ring $\mathbb{Z}[x, y] / J$.

Proof. Define a ring homomorphism by

$$
\begin{aligned}
F: \mathbb{Z}[x, y] & \longrightarrow g \mathcal{R}(\mathcal{C}) \\
x & \mapsto[V(1,0)], \\
y & \mapsto[V(0,1)] .
\end{aligned}
$$

By Lemma 4.3, $F$ is surjective. Moreover, by (1) and (3) of Lemmas 4.1 and 4.3 with $i=0$ and $l=d-1$, it is easy to see that $J \subset \operatorname{ker} F$. So $F$ induces a ring epimorphism:

$$
\begin{aligned}
\bar{F}: \mathbb{Z}[x, y] / J & \longrightarrow g \mathcal{R}(\mathcal{C}) \\
\bar{x} & \mapsto[V(1,0)], \\
\bar{y} & \mapsto[V(0,1)] .
\end{aligned}
$$

Notice that $\left\{\bar{x}^{i} \bar{y}^{j}\right\}_{0 \leq i \leq n-1,0 \leq j \leq d-1}$ is a $\mathbb{Z}$-basis of $\mathbb{Z}[x, y] / J$ hence its $\mathbb{Z}$-dimension is $n d$, which is equal to the $\mathbb{Z}$-dimension of $\mathfrak{R}(\mathcal{C})$. That is, the map $\bar{F}$ is an isomorphism.

We conclude the paper with the following remarks.

Remark 4.5. Keep the same notations $n, d, s, q, Z_{n}$ and $\mathcal{C}(n, s, q)$ as in Section 2.

(1) If one prefers to work on a path algebra rather than a path coalgebra, then one can realize $\mathcal{C}(n, s, q)$ as the representation category of the quasi-Hopf algebra $H(n, s, q)$ which is dual to $M(n, s, q)$. As an algebra, $H(n, s, q)$ is isomorphic to $k Z_{n}^{a} / J^{d}$ where $k Z_{n}^{a}$ is the associated path algebra of the quiver $Z_{n}$ and $J$ is the ideal generated by the set of arrows of $Z_{n}$.

(2) When $s=0$, then $d=$ ord $q$ is a factor of $n$ and $M(n, 0, q)$ is an $n d$-dimensional Hopf algebra. The dual of $M(n, 0, q)$ was considered in [5] by Cibils and the corresponding Clebsch-Gordan formulae were obtained there.

(3) When $s=0$ and ord $q=n$, the algebra $M(n, 0, q)$ is the Taft Hopf algebra [15] whose Green ring $r\left(H_{n}(q)\right)$ (of representation category) was presented in [3]. It is well-known that the Taft algebra is self-dual, hence $q \mathcal{R}(\mathcal{C}(n, 0, q)$ and $r\left(H_{n}(q)\right)$ coincide.

(4) The Green rings $r\left(H_{n, d}\right)$ of the generalized Taft algebras [12] were presented in [14]. It turns out that the representation category of $H_{n, d}$ is a pointed tensor category of finite type, but not connected. The category can be presented as $n / d$ copies of $\mathcal{C}(d, 0, q)$ with $d=$ ord $q$; see [12,13]. The dual of $H_{n, d}$ is exactly $M(n, 0, q)$ with $d=$ ord $q$; see [4]. Thus, the Green ring $\mathcal{Q} \mathcal{R}(\mathcal{C}(n, 0, q))$ is the Green ring of the corepresentation category of $H_{n, d}$, or the Green ring of the Hopf algebra $H_{n, d}^{*}$. As the generalized Taft algebras $H_{n, d}$ are not self-dual if $n \neq d$, so it is natural that $\mathscr{R} \mathcal{R}(\mathcal{C}(n, 0, q))$ and $r\left(H_{n, d}\right)$ are not isomorphic. 


\section{Acknowledgements}

The research of Huang was partially supported by the SDNSF grant 2009ZRA01128 and the IIFSDU grant 2010 TS021. The paper was written while Huang was visiting the University of Antwerp and he is very grateful for its hospitality. He acknowledges the Belgian FWO for the financial support which makes the visit possible.

\section{References}

[1] I. Assem, D. Simson, A. Skowronski, Elements of the Representation Theory of Associative Algebras. Vol. 1. Techniques of Representation Theory, in: London Mathematical Society Student Texts, vol. 65, Cambridge University Press, Cambridge, 2006.

[2] D.J. Benson, Representations and Cohomology: Volume 1, Basic Representation Theory of Finite Groups and Associative Algebras, in: Cambridge Studies in Advanced Mathematics, vol. 30, Cambridge University Press, Cambridge, 1991.

[3] H. Chen, F. Van Oystaeyen, Y. Zhang, The Green rings of Taft algebras, Proceedings of the AMS (2013) in press. arXiv:1111.1837v2 [math.RT]

[4] X.-W. Chen, H.-L. Huang, Y. Ye, P. Zhang, Monomial Hopf algebras, J. Algebra 275 (1) (2004) 212-232.

[5] C. Cibils, A quiver quantum group, Comm. Math. Phys. 157 (1993) 459-477.

[6] C. Cibils, M. Rosso, Hopf quivers, J. Algebra 254 (2) (2002) 241-251.

[7] P. Etingof, S. Gelaki, D. Nikshych, V. Ostrik, Tensor categories, in: Lecture Note for the MIT Course 18.769, 2009. Available at: www-math.mit.edu/ etingof/tenscat.pdf.

[8] P. Etingof, V. Ostrik, Finite tensor categories, Moscow Math. J. 4 (2004) 627-654.

[9] J.A. Green, The modular representation algebra of a finite group, Illinois J. Math. 6 (4) (1962) 607-619.

[10] H.-L. Huang, Quiver approaches to quasi-Hopf algebras, J. Math. Phys. 50 (4) (2009) 043501. p. 9.

[11] H.-L. Huang, From projective representations to quasi-quantum groups, Sci. China Math. 55 (2012) 2067-2080.

[12] H.-L. Huang, H. Chen, P. Zhang, Generalized Taft algebras, Algebra Colloq. 11 (3) (2004) 313-320.

[13] H.-L. Huang, G. Liu, Y. Ye, Quivers, quasi-quantum groups and finite tensor categories, Comm. Math. Phys. 303 (2011) 595-612.

[14] L. Li, Y. Zhang, The Green rings of the generalized Taft algebras, Contemporary Math. 585 (2013) 275-288.

[15] E.J. Taft, The order of the antipode of finite dimensional Hopf algebras, Proc. Natl. Acad. Sci. USA 68 (1971) 2631-2633.

[16] S.J. Witherspoon, The representation ring of the quantum double of a finite group, J. Algebra 179 (1) (1996) 305-329.

[17] S.J. Witherspoon, The representation ring of the twisted quantum double of a finite group, Canad. J. Math. 48 (6) (1996) $1324-1338$.

[18] D. Woodcock, Some categorical remarks on the representation theory of coalgebras, Comm. Algebra 25 (1997) 2775-2794. 